\title{
SPECT/CT technology
}

\author{
P. Ritt $\cdot$ J. Sanders $\cdot$ T. Kuwert
}

Received: 22 July 2014/ Accepted: 15 October 2014/Published online: 31 October 2014

(C) Italian Association of Nuclear Medicine and Molecular Imaging 2014

\begin{abstract}
The continuous development of SPECT over the past 50 years has led to remarkably improved image quality and increased diagnostic confidence. The most influential developments include the realization of hybrid SPECT/CT devices, as well as the implementation of attenuation correction and iterative reconstruction techniques. These developments have led to a preference for SPECT/CT devices over SPECT-only systems and to the widespread adoption of the former, strengthening the role of SPECT/CT as the workhorse of nuclear medicine imaging. New trends in the ongoing development of SPECT/CT are quite diverse. For example, whole-body SPECT/CT images, consisting of acquisitions from multiple consecutive bed positions in the manner of PET/CT, are increasingly performed. Another recent advance has been the incorporation of additional information obtained from the $\mathrm{CT}$ images into the SPECT reconstruction, thereby allowing CT data to be used for more than just attenuation correction and image fusion. Additionally, in recent years, some interesting approaches in detector technology have found their way into commercial products. For example, some SPECT cameras dedicated to specific organs employ semiconductor detectors made of cadmium telluride or cadmium zinc telluride, which have been shown to increase the obtainable image quality by offering a higher sensitivity and energy resolution. Most important, the recent advent of quantitative SPECT/CT which, like PET, can quantify the amount of
\end{abstract}

Color figures online at http://link.springer.com/article/10.1007/ s40336-014-0086-7.

P. Ritt $(\bowtie) \cdot$ J. Sanders $\cdot$ T. Kuwert

Clinic for Nuclear Medicine, University Hospital Erlangen,

Ulmenweg 18, 91054 Erlangen, Germany

e-mail: philipp.ritt@uk-erlangen.de tracer in terms of $\mathrm{Bq} / \mathrm{mL}$ or as a standardized uptake value, is a major innovation that will lead to increased diagnostic accuracy and confidence, especially in longitudinal studies and in the monitoring of treatment response. This article describes some of the physical and technical fundamentals of SPECT/CT and reviews established as well as experimental technical developments. It also introduces the aforementioned recent trends in the field of nuclear medicine imaging, such as specialized collimators, SPECT cameras for anatomical imaging (e.g. cerebral/cardiac imaging) and new detector technologies.

Keywords SPECT $\cdot$ SPECT/CT $\cdot$ Absolute quantification $\cdot$ Multimodal imaging

\section{Introduction}

The steady increase in the number of SPECT/CT devices in European clinics [1], since their commercial introduction in 1999, together with a decrease in the number of SPECTonly devices, are two trends indicative of the considerable confidence that physicians derive from SPECT/CT in clinical practice. The rationale behind this is that the integration of SPECT and CT, through the utilization of techniques like hardware co-registration, attenuation correction and scatter correction, offers numerous diagnostic possibilities.

The individual components of SPECT/CT have a long history. The evolution of SPECT as we know it today involved a multitude of development steps. These include, for example, the invention of the gamma camera [2] in the late 1950 s, early attempts at tomographic imaging involving the use of fixed detectors and rotating chairs [3], the advent of focal plane emission tomography [4] in the 
1960s, and finally the introduction of a system that used rotating gamma cameras [5] in the 1970s. Similarly, the development of the first clinical X-ray CT scanner by Sir G. Hounsfield [6, 7] was necessarily preceded by a number of important achievements, such as the discovery of X-rays by Roentgen in 1895, the mathematical research of Radon [8], and the development of basic CT principles by Oldendorf [9] in the 1960s. A detailed history of SPECT and SPECT/CT can be found in an excellent recent article by Hutton [10].

This article aims to describe some of the physical and technical fundamentals of the field of SPECT/CT and to review established, as well as experimental, technical advances.

In addition, it introduces other recent trends in the field of nuclear medicine imaging, such as specialized collimators, SPECT cameras for anatomical imaging (e.g. cerebral/ cardiac imaging) and new detector technologies.

\section{Technical principle}

The most common SPECT cameras reflect a "one size fits all" approach that aims to address a large range of diagnostic questions relating to various organs of interest and/ or using different radionuclides. The motivation behind this is to ensure that SPECT/CT cameras can be used for as many diagnostic tasks as possible, thus maximizing their cost efficiency.

Most often, this multi-purpose design is achieved by mounting two gamma camera heads on a movable gantry (see Fig. 1). The X-ray transmission device (CT or flatpanel detector) is usually integrated in the same gantry but can often be operated independently. The SPECT and CT acquisitions are, with most devices, carried out sequentially. The SPECT is acquired first, followed by the CT or vice versa. With some systems, the patient needs to be transferred from the SPECT position to the CT position by an axial table movement. However, other systems allow SPECT and CT acquisitions to be performed without the need for table movements [11]. A broad range of transmission devices is available, but, in general, two trends can be identified. The first includes systems featuring fully diagnostic CT devices with fast-rotation $\left(0.5 \mathrm{~s}\right.$ per $\left.360^{\circ}\right)$ detectors that simultaneously acquire data from 16 slices and X-ray tubes with high voltage (up to $140 \mathrm{kVp}$ ), high current (up to $500 \mathrm{~mA}$ ), and automatic exposure control. Often, these systems also support advanced CT capabilities like cardiac gating, calcium scoring and iterative reconstruction. Some research and prototype systems with even higher slice counts (e.g. 64 slices) are available [12]. At the other end of the spectrum there are systems that focus on optimal integration with SPECT by employing co-planar transmission imaging, adapted rotation times (e.g. $12 \mathrm{~s}$ per $360^{\circ}$ ) and dose-optimized acquisition modes with lower tube voltages and currents. SPECT/CT systems with lower CT slice counts (e.g. one or two slices) and decreased temporal resolution are also available. These systems often do not provide the full range of advanced CT features, but still offer cost-efficient attenuation correction and image co-registration.

The gamma cameras themselves usually follow the design of the original Anger camera [2, 13]. First, a projection of the radioactivity distribution is formed via an absorptive collimator mounted on the detectors. A sodium iodide (NaI) scintillator crystal then converts the high energy of the gamma photons into lower energy ultra-violet photons. These are transformed into an electrical signal by photomultipliers. The energy and position of the detected photons is calculated by pulse height spectrometry and by analyzing differences in signals recorded by the photomultipliers, respectively. In recent years, alternatives to the Anger camera have been introduced into clinical practice.

Semiconductive materials like cadmium telluride (CdTe) and cadmium zinc telluride (CZT) convert the gamma photons directly into electrical signals and thus offer better performance when converting gamma photons to electrons; this allows increased energy resolution $(\sim 5$ vs. $\sim 10 \%$ at $140 \mathrm{keV})$ as compared to the Anger technique [14]. Additionally, the linear attenuation coefficient of CZT is slightly higher than that of $\mathrm{NaI}(\sim 3$ vs. $\sim 2 \mathrm{~cm}^{-1}$ at $140 \mathrm{keV}$ ), which could potentially lead to an increased sensitivity [15], although this benefit is often foregone when it is decided, for economic reasons, to limit the thickness of the semiconductors. In fact, the considerable advantages in terms of the photon sensitivity (regularly reported to be 4-8 times higher) and resolution [16] of these cameras are achieved through changes to features other than just the detector material, e.g. through special geometries, adapted collimation and pixelated detectors [17]. For example, for cardiac examination, these advantages allow shorter acquisition times (e.g. 6 vs. 16 min [16]) or considerably diminished patient dose at improved signal-to-noise ratio and image quality (1.15 vs. $2.39 \mathrm{mSv}$ [18]). Unfortunately, the CZT technique is expensive compared to $\mathrm{NaI}$ and photomultipliers, and this restricts its usage to smaller systems that are designed for special applications like cardiac or preclinical imaging [19]. Some prototype systems are also available for imaging of the human brain [20]. With their excellent resolution and sensitivity, CZT systems are particularly suited for scintimammography. In this setting, even lesions with diameters as small as $5 \mathrm{~mm}$ can be reliably detected [21, 22] at significantly reduced patient doses [23].

As regards the movement of the SPECT detectors, modern systems are not limited only to standard $180^{\circ}$ 

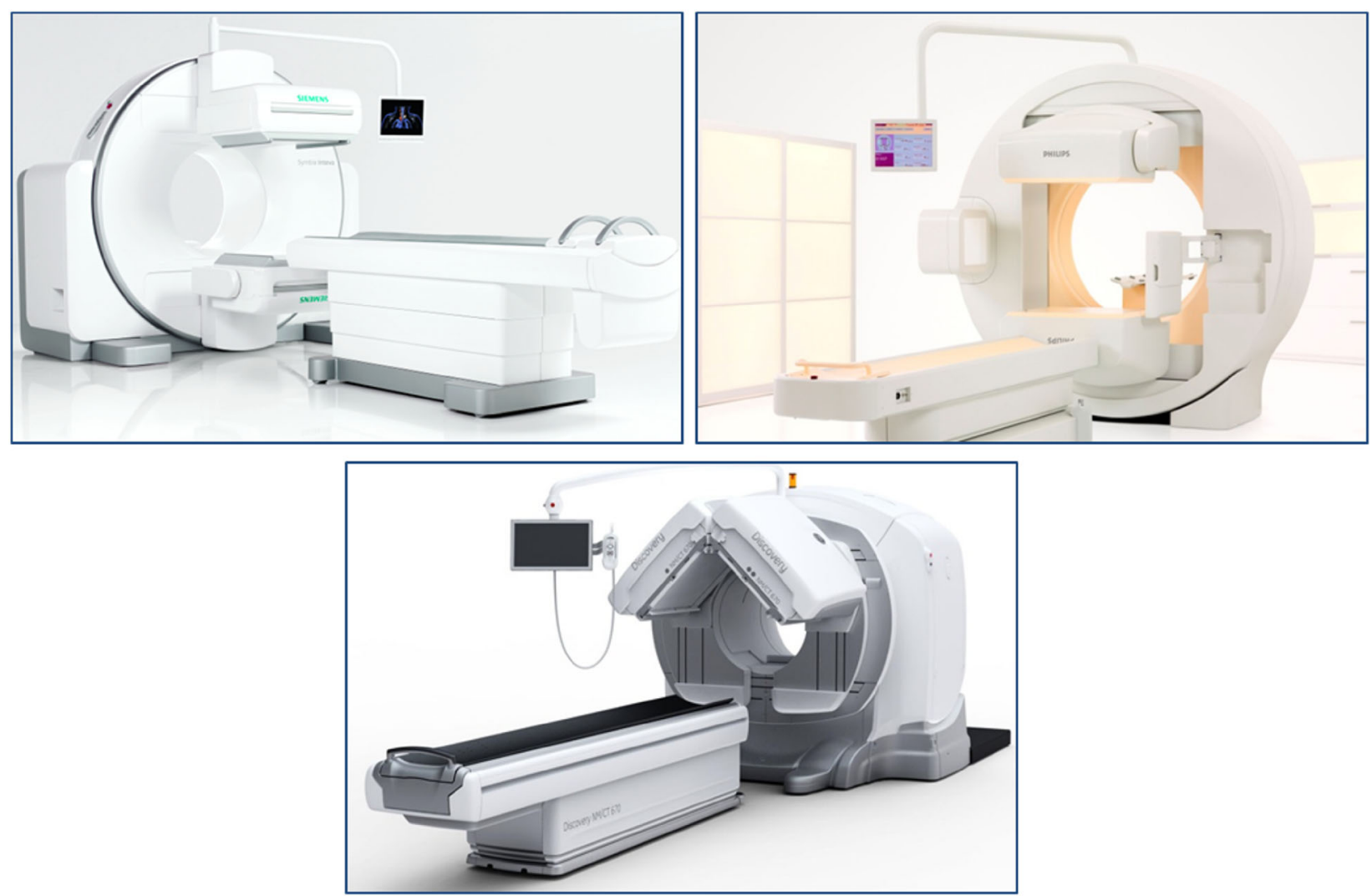

Fig. 1 Examples of SPECT/CT systems. Upper left Siemens Intevo, two gamma camera heads combined with a CT system. Upper right Philips BrightView XCT, two gamma camera heads with a flat-panel detector. Lower image GE Discovery NM/CT 670, two gamma camera heads here configured at $90^{\circ}$ combined with a CT system (color figure online) detector configurations (where the detectors are opposite each other), but also allow other geometries such as $90^{\circ}$ or $76^{\circ}$ for cardiac imaging. Some systems even permit more exotic geometries, where the center of rotation of the detectors can be adjusted individually for each patient. This approach is beneficial if focusing collimators are used since the organ of interest (e.g. the heart) can be kept at the focal point of the acquisition [24]. With this approach, a fourfold increase in sensitivity over parallel-hole collimation is reported, and otherwise unchanged diagnostic information is provided [25]. Another emerging technology in the field of collimators is the application of the (multi-)pinhole principle. Even though such designs offer high spatial resolution compared to parallel-hole collimation, they are rarely used in clinical routine due to their complex imaging characteristics and low sensitivity. However, (multi-)pinhole collimators are regularly applied in small animal imaging [26], where they are able to achieve sub-millimeter spatial resolutions [27].

Prior to the introduction of hybrid SPECT/CT devices, radionuclide transmission measurements (e.g. with Gd-153, Tc-99 m, Ba-133) were commonly employed for the purpose of attenuation correction. However, this method had severe limitations [28], the most important being the relatively poor image quality and high noise at clinically acceptable but still long (5-20 min) measurement times. These limitations, along with the favorable experiences with hybrid PET/CT, led to widespread adoption of SPECT/CT following its introduction in 1999.

\section{SPECT/CT technologies}

\section{Image co-registration}

An important aspect driving the preference for SPECT/CT is the possibility of having co-registered anatomical and functional images, displayed simultaneously in the same image frame. Since SPECT and CT are acquired at the same position or with a known movement of the patient table, the associations of the voxels in the SPECT images with their counterparts in the CT images are generally well defined (hardware registration). This ensures proper global co-registration even when highly organ-specific tracers are used (Fig. 2), although the accuracy of this hardware registration naturally varies for different applications and 
Fig. 2 Labeling of a sentinel lymph node (SLN) using Tc99m Nanocoll. Fused SPECT/ CT (bottom) allows for proper anatomical localization of the SLN, which is beyond the capability of stand-alone SPECT (upper right) and CT (upper left) (color figure online)
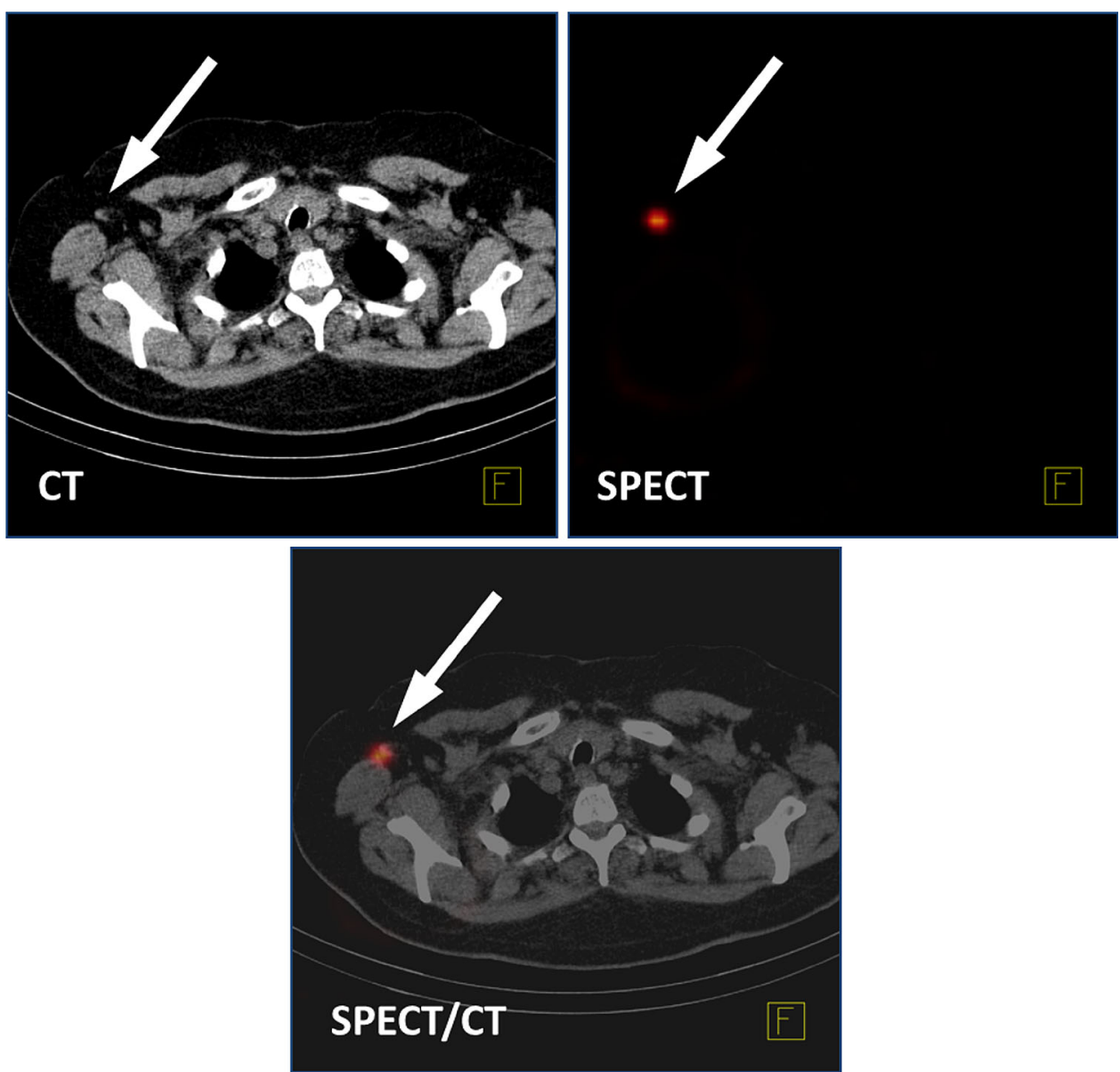

anatomies. In general it can be considered to be accurate to within a few millimeters, e.g. 1-2 $\mathrm{mm}$ for stationary organs [29] and 5-7 $\mathrm{mm}$ for organs that are affected by respiratory motion [30]. One problem that influences the quality of the co-registration is that of the varying time frames over which transmission and emission images are acquired. The acquisition times for emission images are typically in the range of 10-60 min, whereas times of below one min are normal for CT images. This can lead to misalignments, as the SPECT image is the result of data averaged over several breathing and heartbeat cycles, whereas the CT image is almost movement free. Some techniques can be used to reduce artifacts caused by this discrepancy, such as acquiring the CT data in a partial expiratory state [31] or acquiring the SPECT images in gated fashion. Furthermore, any patient movement occurring between the acquisition of SPECT and CT could naturally also lead to artifacts. In this case, manual post registration may help to reduce the extent of any misregistration [32].

Nevertheless, despite possible artifacts, it has been clinically established that the multimodal information from co-registered SPECT and CT provides physicians with useful additional diagnostic information [33, 34].

\section{Attenuation correction with CT}

Another advantage of SPECT/CT is its ability to correct the SPECT images for photon attenuation in a fast and accurate manner. This is the most important correction, as it allows quantitatively accurate SPECT/CT, in other words reconstructed images with quantification in terms of absolute units of $\mathrm{Bq} / \mathrm{mL}$ or some equivalent [35].

Photon attenuation occurs when a photon emitted in the direction of a detector does not reach the detector due to interaction with matter. The predominant type of interaction at clinically relevant photon energies is called the Compton effect. This term refers to the scattering of photons upon collision with quasi-free electrons and the phenomenon is heavily dependent on the electron density of the material being traversed. A CT device provides a direct measurement of the attenuating properties of the specimen of interest. In practice, however, the CT images are usually reconstructed in Hounsfield units which subsequently need to be converted into linear attenuation coefficients (units of $1 / \mathrm{cm}$ ) at the respective photon energy of the radionuclide.

The attenuating properties at location $\mathbf{x}$ for photons of a certain energy $E$ are given by the linear attenuation 
Fig. 3 Bilinear conversion of Hounsfield units into linear attenuation coefficients for different photon energies. The attenuation coefficients are smaller with increasing photon energies
Conversion of HU to Linear Attenuation Coefficients

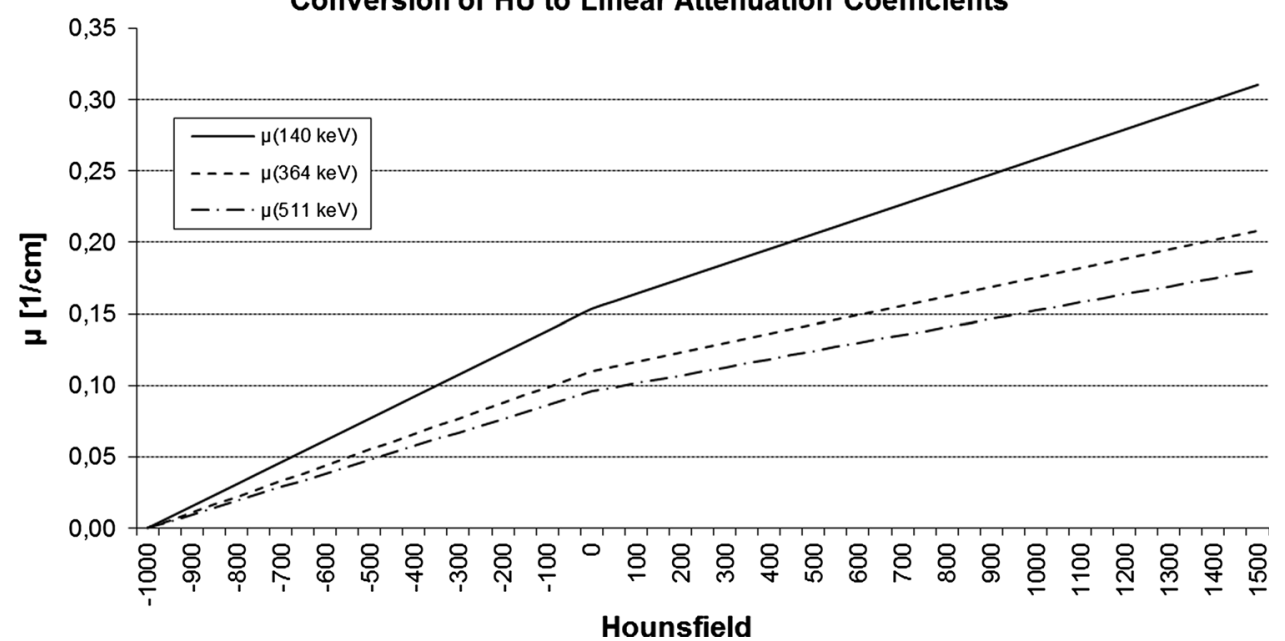

coefficient $\mu(E, \mathbf{x})$. In most cases, a bi-linear transformation (Fig. 3) is applied. The parameters of the transformation (slope and intercept) are defined by the linear attenuation coefficients ( $\mu$-values) for air, bone and water at the respective photon energies. Additionally, the transformation depends on the effective energy $E_{\mathrm{CT}}$ of the CT and hence is specific to the selected acceleration voltage.

Interestingly, due to this transformation and due to the fact that the CT images are often down-sampled to lower resolutions for attenuation correction, even visually very noisy (low-dose, $110 \mathrm{kVp}+12 \mathrm{mAs}$ ) CT acquisitions cause negligible $(<3 \%)$ inaccuracies in the reconstructed SPECT images [36].

However, despite this reliability, the bilinear transformation method can lead to errors in terms of quantitative accuracy. This may occur, for example, if assumptions about either the energy of the radionuclide or the effective energy of the CT scanner are incorrect, or if CT beamhardening artifacts are not corrected.

An additional source of errors is the presence of dense material in the field of view of the CT device. For example, metallic implants like pacemakers or prostheses can cause artifacts and lead to bias in the attenuation map [37]. Consequently, these artifacts are transferred into the attenuation-corrected SPECT images and result in erroneous radioactivity concentrations. Similar degrading effects are reported with the use of CT contrast agents [38] in SPECT/CT acquisitions. For this reason, studies that rely on exact quantification should avoid the use of contrastenhanced CT images for attenuation correction. Furthermore, for organs which are affected by motion, a mismatch between the emission and the transmission scan will likely introduce errors into the attenuation-corrected SPECT scan. This is very frequently the case in organs like the heart, the lung, and the abdominal organs [39]. Often, the bias can be reduced by performing a re-registration before the reconstruction of the attenuation-corrected images [40]. Results from the literature indicate that acquiring the CT in a certain respiratory phase or calculating CT images that are averaged over several respiratory phases will also diminish the potential bias [41]. Other options include various approaches to respiratory gating [42] or motion correction of SPECT data [43, 44].

\section{Scatter correction}

Scatter, which is related to attenuation, is an important factor which degrades the quality of SPECT images, although its correction is somewhat less important [45] than the corrections for the point spread function (to be introduced in the section Image reconstruction) or for photon attenuation. However, since very accurate technical solutions for these latter problems are already available in clinical practice, scatter remains a major limiting factor of the image quality and quantitative accuracy of current SPECT systems, particularly when using isotopes with complex emission spectra. There exist numerous techniques for scatter correction; describing them all is beyond the scope of this article, but an extensive review can be found in [46].

As mentioned above, the Compton effect is the dominant process for scattering of photons in the patient and in the collimators at clinically relevant energies. Coherent scattering, an interaction between the photons and atoms or molecules, which is another phenomenon capable of deflecting photons from their original direction, is important only for energies below $50 \mathrm{keV}$. With Compton scattering, the photon transfers part of its energy to an electron and should be detected at lower energies in an ideal detector with perfect energy resolution. In a real detector, the uncertainty of energy determination limits the 


\section{Energy of Photons Undergoing Compton Scatter}

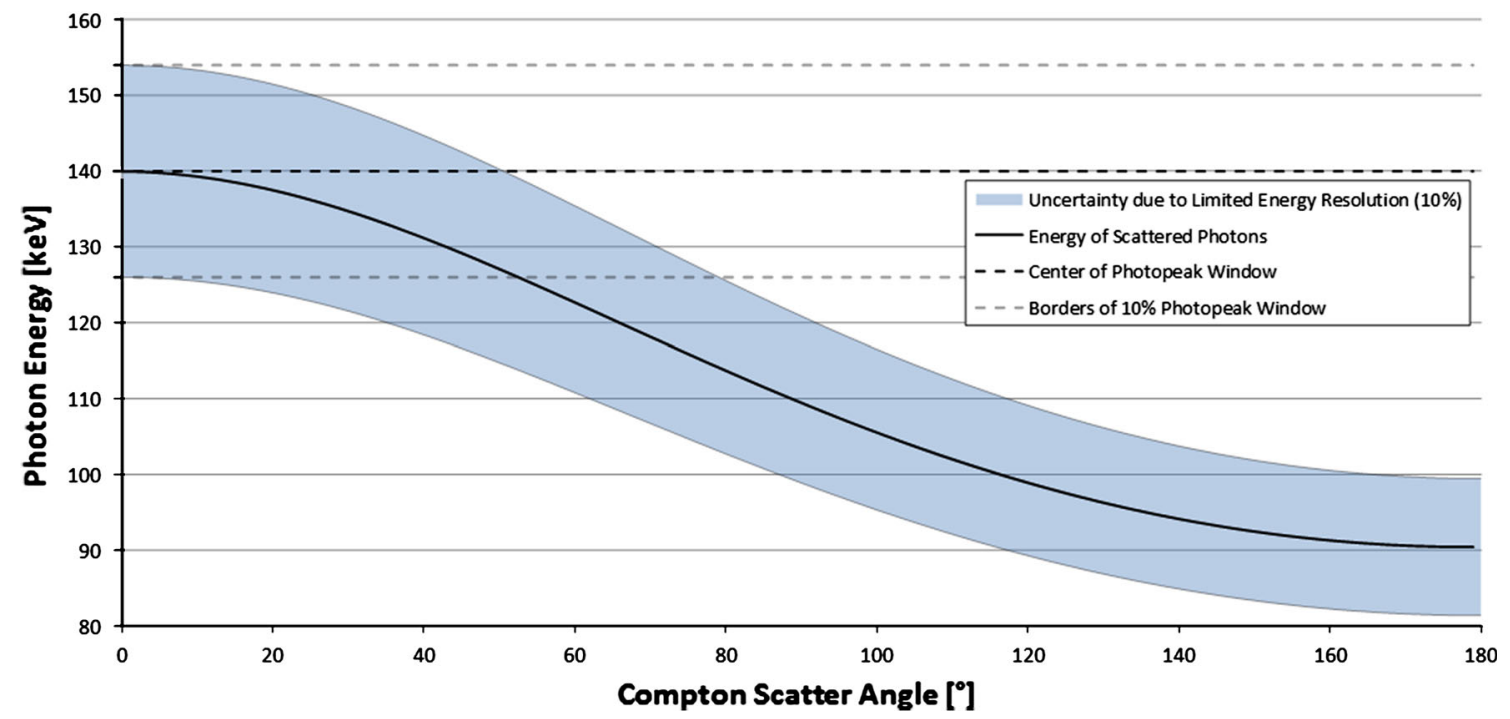

Fig. 4 Energy of Tc-99m photons after undergoing Compton scattering at a certain angle. The dashed lines represent the typical $20 \%$ photopeak window that is applied for acquiring data. The shaded area represents the uncertainty in the determination of the

detectable energy loss. In general, the amount of energy loss depends on the scatter angle. The result of this relationship is that scattered photons are often detected at locations unrelated to their initial trajectories, leading to a loss of information about the activity distribution being imaged. The proportion of scattered photons in a particular acquisition is object dependent but can be rather high. One study reports that the ratio of scattered to unscattered photons in myocardial Tc-99 $\mathrm{m}$ imaging is typically 0.34 [47]. The amount of Compton scatter originating in the collimators is most likely negligible for $140 \mathrm{keV} \mathrm{Tc}-99 \mathrm{~m}$ imaging but increases with increasing energy and thus is considerably higher for I-131 at $364 \mathrm{keV}$ [48]. In order to minimize the amount of scatter and to exclude photons that do not originate from the decay of the respective radionuclide, energy windows around the photopeak of interest are applied. Nevertheless, due to the limited energy resolution of SPECT detectors and the typical width of the photopeak windows, a considerable amount of scattered photons is still recorded, as seen as in Fig. 4.

Figure 4 depicts the dependence of the energy of scattered photons (black line) on the scatter angle. The shaded area indicates the uncertainty of the energy measurement of the SPECT detectors, which is typically around $10 \%$ for NaI. The dashed lines indicate a $20 \%$ energy window around the $140 \mathrm{keV}$ Tc-99 m photopeak. We find that even photons that were scattered by 80 degrees are still regularly included in the SPECT photopeak projections.

This underlines the fact that additional techniques are necessary to account and correct for scatter. First, the energy of the photons which is assumed to be $10 \%$, similar to $\mathrm{NaI}$ detectors. Even relatively large scatter angles of 60-80 degrees still are likely to be detected in the photopeak window (color figure online)

amount of scattered photons in the photopeak window needs to be estimated. Here, two paradigms are possible: estimates based on directly measured data or estimates based on modeling. The most common approach, i.e. that of measuring the counts in multiple additional energy windows $[49,50]$ adjacent to the photopeak window, belongs to the former paradigm. For the triple energy window (TEW) technique, the scattered photons in the photopeak can be calculated according to Eq. 1 for every pixel. Here, $n_{s}$ are the counts of the pixel that were scattered and $n_{l}, n_{u}$ are the measured counts in the lower and upper scatter windows. The widths of the photopeak, the lower scatter, and upper scatter window are denoted by $w_{p}$, $w_{l}$, and $w_{u}$.

$n_{s}=\left(\frac{n_{l}}{w_{l}}+\frac{n_{u}}{w_{u}}\right) \times \frac{1}{2} w_{p}$

The calculated amount of scattered photons is then incorporated into the image reconstruction. This can be achieved in multiple ways. The simplest approach is to subtract the counts from the photopeak window. However, it was found [51] that incorporating the amount of scatter directly into the forward projector in the maximum likelihood expectation maximization (MLEM) algorithm is a superior approach in terms of image quality and quantitative accuracy. This is to a large extent explained by the high amount of noise in the scatter window data which is due to the generally low count number in these windows.

As an extension of the TEW method, so-called spectral models can be applied. Such methods try to improve the 
accuracy and the noise properties of the scatter estimates via a finer sampling of the energy distribution of the photons that reach each detector pixel. Ideally, one would record the energy of each photon individually (list-mode acquisition). With this information, models that predict the amount of scatter in the photopeak window could be estimated with good confidence and low noise [52]. Despite the high accuracy of these methods, to the best of our knowledge, no scientific studies in this field have been published in recent years. However, we assume that such approaches might attract renewed interest if list-mode acquisitions become routinely available on commercial SPECT systems.

Scatter estimation and correction with the help of full Monte Carlo (MC) simulations has been a trend in research in recent years. These simulations are supported by the availability of faster computational hardware and the development of accelerated versions of the MC algorithms [53]. Two important prerequisites for these simulations are the estimates of the spatial distributions of the radioactive source and of the probability that scatter occurs. For this reason, MC simulations are usually carried out in each step of the iterative SPECT reconstruction, since the source distribution is not known a priori, and is therefore updated at each iteration [54]. The probability that scatter occurs can be estimated using transmission scans of the object, which are readily available with SPECT/CT systems. Drawbacks of these methods are the still comparatively long calculation times and the inability to account for photons that originate from outside the camera's field of view. A faster method that relies on partial MC simulations and approximated scatter patterns is called effective source scatter estimation [55].

However, despite the availability of very sophisticated scatter correction methods, the estimation of scatter by either TEW or DEW (the dual energy window approach that neglects upper scatter) and its correction in the MLEM or in the ordered subset expectation maximization (OSEM) algorithm is still the standard approach in clinical SPECT.

\section{Multimodal image reconstruction and resolution recovery}

Image reconstruction is the technique used to transform the measured 2-D projection data into a 3-D activity distribution. In contrast to $\mathrm{CT}$, which often employs analytic techniques like back projection, iterative reconstruction techniques prevail in SPECT. For a review of the different algorithms, see [56]. For an overview of the reconstructed resolution according to the NEMA NU-2007 standard calculated using filtered back projection (FBP), see Table 1.
Table 1 Typical values for the reconstructed SPECT spatial resolution and volume sensitivity, as reported by the manufacturers and if available to the authors

\begin{tabular}{|c|c|c|c|}
\hline $\begin{array}{l}\text { System (all } \\
\text { with LEHR) }\end{array}$ & $\begin{array}{l}\text { Siemens } \\
\text { Symbia } \\
\text { Intevo }\end{array}$ & $\begin{array}{l}\text { Philips } \\
\text { BrightView } \\
\text { XCT }\end{array}$ & $\begin{array}{l}\text { GE Discovery } \\
\text { NM/CT } 670\end{array}$ \\
\hline \multicolumn{4}{|c|}{ Reconstructed resolution } \\
\hline $\begin{array}{l}\text { Central } \\
\text { transaxial }\end{array}$ & $\leq 10.2 \mathrm{~mm}$ & $\leq 10.3 \mathrm{~mm}$ & $\leq 9.9 \mathrm{~mm}$ \\
\hline $\begin{array}{l}\text { Peripheral } \\
\text { radial }\end{array}$ & $\leq 9.8 \mathrm{~mm}$ & $\leq 10.5 \mathrm{~mm}$ & $\leq 9.9 \mathrm{~mm}$ \\
\hline $\begin{array}{l}\text { Peripheral } \\
\text { tangential }\end{array}$ & $\leq 8.4 \mathrm{~mm}$ & $\leq 9.0 \mathrm{~mm}$ & $\leq 7.5 \mathrm{~mm}$ \\
\hline \multicolumn{4}{|c|}{ Volume sensitivity } \\
\hline $\begin{array}{l}\text { UFOV, per } \\
\text { axial } \mathrm{cm}\end{array}$ & $\begin{array}{c}12,000(\mathrm{cts} / \mathrm{sec}) / \\
\left(\mathrm{MBq} / \mathrm{cm}^{2}\right)\end{array}$ & $\begin{array}{c}10,000(\mathrm{cts} / \mathrm{sec}) / \\
\left(\mathrm{MBq} / \mathrm{cm}^{2}\right)\end{array}$ & n.a. \\
\hline
\end{tabular}

LEHR low-energy high-resolution collimator, UFOV useful field of view

Most iterative techniques rely on mathematical models which assume a linear dependency of the image $\mathbf{f}$ (vector with elements $f_{j}$ representing the voxels of the activity distribution) and the projection data $\mathbf{p}$ (vector with elements $p_{i}$ representing detector pixels) via the system matrix A. Each element $A_{i j}$ describes the probability that a photon originating from voxel $j$ is detected at pixel $i$. The projection data thus can be expressed as a multiplication of the system matrix $\mathbf{A}$ by $\mathbf{f}$ (Eq. 2):

Af $=p$

The most common iterative techniques are the MLEM algorithm and its accelerated version, OSEM. MLEM was first applied to SPECT in 1984 [57, 58]. Its relatively high computational requirements (compared with filtered back projection) delayed its widespread use until the late 1990s. It is based on the fact that the image $\mathbf{f}$ consists of Poissondistributed random variables and it seeks to maximize the likelihood of the observed data given the source $\mathbf{f}$. The well-known update rule of the iterative ML-EM algorithm is given in Eq. 3 .

$f_{j}^{\text {new }}=\frac{f_{j}^{\text {old }}}{\sum_{l} a_{l j}} \sum_{i} a_{i j} \frac{p_{i}}{\sum_{k} a_{i k} f_{k}^{\text {old }}}$

Besides explicit Poisson-noise modeling, an important additional advantage of MLEM is its capacity to implement the crucial attenuation and scatter corrections and to model the collimator-detector response (CDR) directly into the reconstruction. While the attenuation and scatter corrections model the interaction of the photons with the imaged object, the latter represents their interaction with the detection system (collimator, crystal and electronics). The most important components of the CDR are: (a) the intrinsic response that is caused by scatter occurring in the 

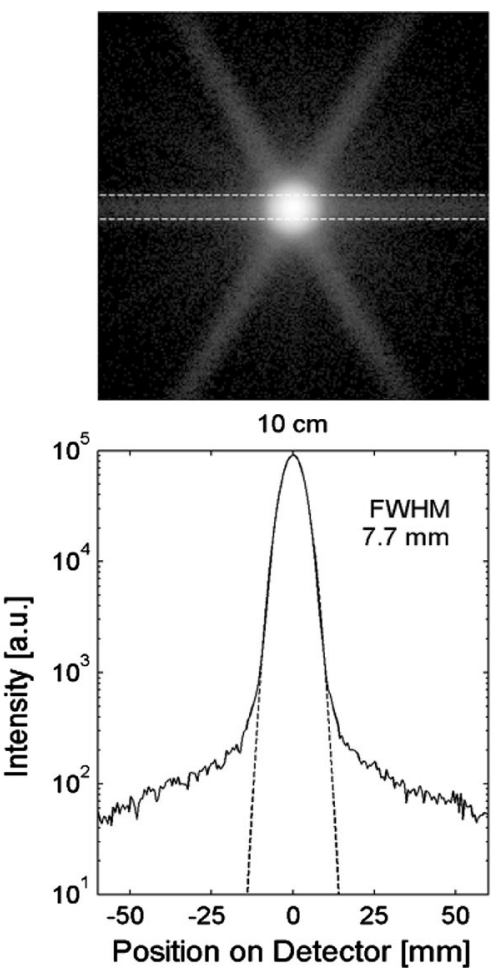

Fig. 5 Top row images of Tc-99m point sources measured at different typical source-to-collimator distances. Bottom row line profiles through the center of the point sources. The extent of the line profiles is indicated in the upper left image. Gaussian functions were fitted to the line profiles. The full widths at half maximum (FWHM) values of the Gaussians are provided as well. The FWHM increases

crystal itself and the uncertainty of the position estimation of the detected photon, (b) the geometric response which represents a distance-dependent spatial resolution caused by the finite bore length and width of the collimator, (c) scatter in the collimator itself, and (d) septal penetration when photons are detected after passing through the walls of the collimator holes. $(\mathrm{a}-\mathrm{c})$ can be seen in the bright central region of Fig. 5, which shows point sources taken at different source-to-collimator distances. Upon closer examination, faint star-shaped structures become visible. The geometry of these structures depends on the shape of the holes (hexagonal in this example). With increasing source-to-collimator distances, the counts are distributed over wider areas and the stars appear to be dilated. In order to obtain quantitatively correct images, these effects need to be taken into account as accurately as possible. The CDR is commonly assumed to be Gaussian and modeled as such in the reconstruction. In general, it is reported that this leads to significantly improved image quality [59-61]. The importance of CDR compensation was shown in a large multi-center study [62] that compared a variety of advanced SPECT/CT reconstruction algorithms for cardiac phantoms and found considerable improvements in image with increasing source-to-collimator distance. Additionally, it is found that the center of the point sources is sufficiently approximated by the Gaussian. However, data taken at greater distance are heavily underestimated by the model. Please note that the images of the point sources and the line profiles are displayed in logarithmic units for better visualization

quality. The study used the full width at half maximum (FWHM) of the wall thickness of the left ventricle as a measure of the spatial resolution and reported a significant reduction relative to FBP (on average $22.0 \mathrm{~mm}$ FWHM) for OSEM without CDR modeling (20.6 mm FWHM) and with CDR modeling and corrections for scatter $(16.2 \mathrm{~mm}$ FWHM). Similar trends in favor of the advanced reconstruction techniques are reported for other image quality metrics like contrast and background homogeneity. Additionally, several studies found that with iterative reconstruction techniques, the acquisition time or patient dose could be reduced by a factor of up to two, for example in cardiac [63, 64] and skeletal examinations [65].

Still, it is clear that the Gaussian modeling approach has only limited precision since significant differences between the model and the real CDR can be found, especially in regions on the edge of the geometric response. There, the Gaussian model systematically underestimates the number of detected counts (see bottom of Fig. 5). Additionally, a rotationally invariant function like the Gaussian cannot express the contours found in real CDRs which mimic the shape of the collimator bore. Multiple solutions have been proposed for further improving the modeling: $\mathrm{MC}$ 
Fig. 6 Sagittal slices, 63-yearold female patient, $454 \mathrm{MBq}$ Tc-99 m-bisphosphonate, suspected arthritis. Left Siemens xSPECT Bone reconstruction. Information from $\mathrm{CT}$ is used in order to improve the SPECT reconstruction. Center OSEM 3D reconstruction. Right FBP reconstruction of the low-dose CT (color figure online)
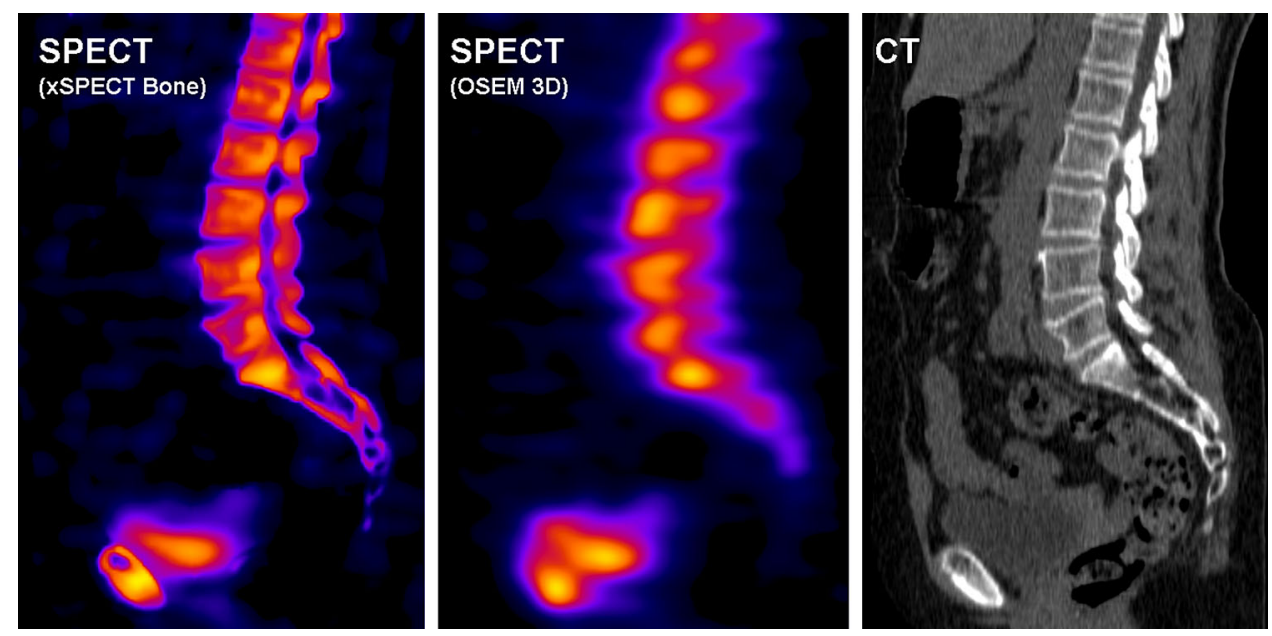

simulations can be used to calculate the CDR during reconstruction [66] or prior to reconstruction [67]. In later approaches, a set of distance-dependent CDRs is calculated, stored and then used in the iterative reconstruction. Other approaches rely on more sophisticated mathematical models and fit their free parameters to a set of point source measurements [68]. Nevertheless, Gaussian modeling is the method that is applied in most clinically available tools.

In addition to OSEM, other reconstruction methods like the weighted least squares conjugate gradient (WLS-CG) may be used. Traditional methods may also be extended by the addition of extra information meant to, for example, improve resolution or reduce noise. One embodiment of such approaches is the family of maximum a posteriori methods (see Eq. 4), where a penalty function $U$ containing prior information may be added to the MLEM formulation. This is a useful extension, as the traditional SPECT reconstruction often suffers from low resolution and high image noise, particularly at high iteration numbers. In the penalty function, prior knowledge derived from anatomical images (CT or MRI) can be implemented [69].

$f_{j}^{\text {new }}=\frac{f_{j}^{\text {old }}}{\sum_{l} a_{l j}} \sum_{i} a_{i j} \frac{p_{i}}{\sum_{k} a_{i k} f_{k}^{\text {old }}+\beta \frac{\partial U}{\partial f_{j}^{\text {new }}}}$

Similarly, a new reconstruction method (xSPECT Bone, Siemens Healthcare) expands upon WLS-CG and integrates information from $\mathrm{CT}$ for much more than simple attenuation correction [70]. Here, the use of CT data aims to improve the spatial resolution of reconstructed SPECT images and to obtain higher quantitative accuracy (Fig. 6). This is achieved by segmenting the CT image into different tissue classes and by assuming that the relative amount of Tc-99 $\mathrm{m}$ diphosphonate uptake is similar within each class. Effectively, the CT image is used to aid in the delineation of certain boundaries of the activity distribution. To the best of our knowledge, only a pilot study has been performed to systematically evaluate the amount of image quality improvement obtained with this method [71]. However, more work in this area is expected to be forthcoming.

\section{Quantitative SPECT/CT imaging}

Absolute quantification in terms of image units in $\mathrm{Bq} / \mathrm{mL}$ or SUV is the standard in PET imaging. Although some reports suggested that SPECT is not a quantitative modality $[72,73]$, technological advances have led to an increase in the number of papers reporting high quantitative accuracy with SPECT, mainly evaluated in phantom or simulation studies.

For example, Shcherbinin et al. [74] reported errors of between $3 \%$ and $5 \%$ in a study on a torso phantom using the isotopes Tc-99 m, I-123, I-131 and In-111. Du et al. [75] achieved a $2 \%$ error for I-123 in a brain phantom. Vandervoort et al. [76] performed simulation studies on the Mathematical Cardiac Torso phantom and on a Tc-99 mfilled torso phantom and reported $8 \%$ error for the simulation and within $4 \%$ error for the phantom study. Da Silva et al. [77] reported $8 \%$ error in an anthropomorphic phantom with cardiac insert, for the isotope Tc-99 m. For the in vivo quantification of Tc-99 $\mathrm{m}$ in 16 patients, Zeintl et al. [78] applying corrections for point spread function, attenuation and scatter, found average deviations of $6.8 \%$ between the activity concentration obtained on SPECT and a well counter measurement of the activity concentration in the bladder. Using the same methods, Cachovan et al. [79] reported uptake of Tc-99 m-bisphosponate in the lumbar spine in 50 patients.

The quantification of diagnostic tracers is of great interest if one's aim is to evaluate the course of a disease. Another potential application in this field is the quantification of therapeutic radionuclides for dosimetric purposes. In this regard, Sherbinin et al. [80] reported a $14.2 \%$ 
overestimation of the activity concentration of a $70 \mathrm{~mL}$ container filled with Lu-177. Similarly, Beauregard et al. [81] obtained a deviation of 7-9 \% for Lu-177 in a phantom with cylindrical compartments, applying corrections for PSF, attenuation, scatter and dead-time. For the in vivo quantification, they reported an average error of $3.1 \%$ for six patients. Unfortunately larger studies that evaluate the quantitative accuracy of today's SPECT systems for therapeutic tracers are still lacking. Nevertheless, some manufacturers have recently started offering SPECT systems that generate quantitative images for a limited number of isotopes.

\section{Concluding discussion}

The continuous development of SPECT and SPECT/CT over the past 50 years has led to remarkable improvements in image quality and diagnostic confidence. The most influential developments include, to name but a few, the realization of hybrid SPECT/CT devices, the implementation of attenuation correction, and iterative reconstruction techniques.

In recent years, some interesting approaches in detector technology have found their way into commercial products. For example, some SPECT cameras dedicated to specific organs use semiconductive materials like CZT as detector material. It has been shown that this could help to further increase the obtainable image quality.

New trends in the ongoing development of SPECT/CT are quite diverse. For example, whole-body SPECT/CT images consisting of acquisitions from multiple consecutive bed positions, similar to PET/CT, have begun to increase. Yet the relatively long SPECT acquisition times (typically $15 \mathrm{~min}$ per bed position and $30-45 \mathrm{~min}$ for the trunk), together with the increased radiation dose from the extended CT field of view, are likely to hamper the routine application of these acquisitions in clinical practice. Even though new reconstruction techniques already allow for acquisition time reduction, in the future, new collimator and detector designs with higher photon sensitivity could further support this development. Additionally, this trend could be facilitated by the incorporation of low-dose concepts applicable to CT like, for example, the use of iterative CT reconstruction in SPECT/CT.

As with PET, the availability of SPECT list-mode data could allow an enormous gain of information. This information could be used to improve estimation and correction of confounding factors like patient motion [42] and scattered radiation [82]. Unfortunately, to the best of our knowledge, such techniques are so far available only for research and not for clinical use.
Another recent development has been the incorporation of additional information obtained from the $\mathrm{CT}$ images into the SPECT reconstruction, in other words the use of CT data for more than just attenuation correction and image fusion. This development could lead to improved spatial resolution and reduced partial volume effects. This technique has been commercially introduced for Tc-99 m bone scans only. However, it could be extremely useful with other tracers as well.

Another important aspect of SPECT/CT is the fact that the technique, like PET, allows quantification of the amount of tracer in terms of $\mathrm{Bq} / \mathrm{mL}$ or as a SUV. Numerous publications have shown that absolute quantification is feasible $[78,79,83,84]$. This can be expected to further boost diagnostic confidence, e.g. for judging the efficacy of a treatment through follow-up acquisitions from the same patient or for improving dosimetry for radionuclide therapy [81].

The ever-increasing number of publications on SPECT/ CT show that this modality is of great clinical interest. New technical developments will strengthen this trend and ensure that SPECT/CT remains the workhorse of nuclear medicine, even when compared to other strong contenders like PET/CT or PET/MRI.

Conflict of interest Philipp Ritt declares that he has no conflict of interest. Dr. Kuwert occasionally gives lectures for Siemens Healthcare. The Clinic of Nuclear Medicine of the University Hospital Erlangen has a research cooperation with Siemens concerning the development and validation of SPECT/CT technologies.

Human and animal studies This article does not contain any studies with human or animal subjects performed by any of the authors.

\section{References}

1. European Association of Nuclear Medicine (2010) Status of Nuclear Medicine in Europe. www.kfnm.dk/div/Results_EANM Survey_2010.pdf. Accessed 10 October 2014

2. Anger HO (1958) Scintillation camera. Rev Sci Instrum 29:27-33

3. Anger HO, Price DC, Yost PE (1967) Transverse-section tomography with the scintillation camera. J Nucl Med 8:314-315

4. McAfee JG, Mozley JM, Stabler EP (1969) Longitudinal tomographic radioisotopic imaging with a scintillation camera: theoretical considerations of a new method. J Nucl Med 10:654-659

5. Jaszczak RJ, Murphy PH, Huard D, Burdine JA (1977) Radionuclide emission computed tomography of the head with ${ }^{99} \mathrm{mTc}$ and a scintillation camera. J Nucl Med 18:373-380

6. Richmond C (2004) Sir Godfrey Hounsfield BMJ 329:687. doi:10.1136/bmj.329.7467.687

7. Hounsfield GN (1973) Computerized transverse axial scanning (tomography): part 1 Description of system. Br $\mathrm{J}$ Radiol 46:1016-1022. doi:10.1259/0007-1285-46-552-1016

8. Radon J (1986) On the determination of functions from their integral values along certain manifolds. IEEE Trans Med Imaging 5:170-176. doi:10.1109/TMI.1986.4307775 
9. Oldendorf WH (1961) Isolated flying spot detection of radiodensity discontinuities - displaying the internal structural pattern of a complexobject. Ire Trans Biomed Electron. BME-8:68-72. doi:10.1109/TBMEL.1961.4322854

10. Hutton BF (2014) The origins of SPECT and SPECT/CT. Eur J Nucl Med Mol Imaging 41:3-16. doi:10.1007/s00259-013-2606-5

11. Sowards-Emmerd D, Balakrishnan K, Wiener J, Shao L, Ye J (2009) CBCT-subsystem performance of the multi-modality Brightview XCT system (M09-26). IEEE Nucl Sci Symp Conf Rec 3053-3058

12. Buck AK, Nekolla S, Ziegler S, Beer A, Krause BJ, Herrmann K, Scheidhauer K, Wester H-J, Rummeny EJ, Schwaiger M, Drzezga A (2008) SPECT/CT. J Nucl Med 49:1305-1319. doi:10.2967/ jnumed.107.050195

13. Anger HO (1964) Scintillation camera with multichannel collimators. J Nucl Med 5:515-531

14. Bocher M, Blevis IM, Tsukerman L, Shrem Y, Kovalski G, Volokh L (2010) A fast cardiac gamma camera with dynamic SPECT capabilities: design, system validation and future potential. Eur J Nucl Med Mol Imaging 37:1887-1902. doi:10.1007/ s00259-010-1488-z

15. Cherry SR, Sorenson JA, Phelps ME (2003) Physics in Nuclear Medicine, 3rd edn. Elsevier, Philadelphia

16. Imbert L, Poussier S, Franken PR, Songy B, Verger A, Morel O, Wolf D, Noel A, Karcher G, Marie PY (2012) Compared performance of high-sensitivity cameras dedicated to myocardial perfusion SPECT: a comprehensive analysis of phantom and human images. J Nucl Med 53:1897-1903. doi:10.2967/jnumed. 112.107417

17. Slomka PJ, Berman DS, Germano G (2014) New cardiac cameras: single-photon emission CT and PET. Semin Nucl Med 44:232-251. doi:10.1053/j.semnuclmed.2014.04.003

18. Einstein AJ, Blankstein R, Andrews H, Fish M, Padgett R, Hayes SW, Friedman JD, Qureshi M, Rakotoarivelo H, Slomka P, Nakazato R, Bokhari S, Di Carli M, Berman DS (2014) Comparison of image quality, myocardial perfusion, and left ventricular function between standard imaging and single-injection ultra-low-dose imaging using a high-efficiency SPECT camera: the MILLISIEVERT study. J Nucl Med. doi:10.2967/jnumed.114.138222

19. Tan J-W, Cai L, Meng L-J (2009) A prototype of the MRIcompatible ultra-high resolution SPECT for in vivo mice brain imaging. IEEE Nucl Sci Symp Conf Rec 2800-2805. doi:10. 1109/NSSMIC.2009.5401656

20. Suzuki A, Takeuchi W, Ishitsu T, Tsuchiya K, Morimoto Y, Ueno Y, Kobashi K, Kubo N, Shiga T, Tamaki N (2013) Highsensitivity brain SPECT system using cadmium telluride (CdTe) semiconductor detector and 4-pixel matched collimator. Phys Med Biol 58:7715-7731. doi:10.1088/0031-9155/58/21/7715

21. Hruska CB, Weinmann AL, O'Connor MK (2012) Proof of concept for low-dose molecular breast imaging with a dual-head CZT gamma camera. Part I Evaluation in phantoms. Med Phys 39:3466-3475. doi:10.1118/1.4718665

22. Brzymialkiewicz CN, Tornai MP, McKinley RL, Bowsher JE (2005) Evaluation of fully 3-D emission mammotomography with a compact cadmium zinc telluride detector. IEEE Trans Med Imaging 24:868-877

23. Hruska CB, Weinmann AL, Tello Skjerseth CM, Wagenaar EM, Conners AL, Tortorelli CL, Maxwell RW, Rhodes DJ, O'Connor MK (2012) Proof of concept for low-dose molecular breast imaging with a dual-head CZT gamma camera. Part II Evaluation in patients. Med Phys 39:3476-3483. doi:10.1118/1.4719959

24. Rajaram R, Bhattacharya M, Xinhong D, Malmin R, Rempel TD, Vija AH, Zeintl J (2011) Tomographic performance characteristics of the IQ-SPECT system. IEEE Nucl Sci Symp Conf Rec 2451-2456. doi:10.1109/NSSMIC.2011.6152666
25. Pirich C, Keinrath P, Barth G, Rendl G, Rettenbacher L, Rodrigues M (2014) Diagnostic accuracy and functional parameters of myocardial perfusion scintigraphy using accelerated cardiac acquisition with IQ SPECT technique in comparison to conventional imaging. Q J Nucl Med Mol Imaging 2014 Jun 30 (Epub ahead of print)

26. Higaki Y, Kobayashi M, Uehara T, Hanaoka H, Arano Y, Kawai $\mathrm{K}$ (2013) Appropriate collimators in a small animal SPECT scanner with CZT detector. Ann Nucl Med 27:271-278. doi:10. 1007/s12149-012-0681-5

27. Boisson F, Zahra D, Parmar A, Gregoire MC, Meikle SR, Hamse H, Reilhac A (2013) Imaging capabilities of the Inveon SPECT system using single-and multipinhole collimators. J Nucl Med 54:1833-1840. doi:10.2967/jnumed.112.117572

28. O'Connor MK, Kemp B, Anstett F, Christian P, Ficaro EP, Frey E, Jacobs M, Kritzman JN, Pooley RA, Wilk M (2002) A multicenter evaluation of commercial attenuation compensation techniques in cardiac SPECT using phantom models. J Nucl Cardiol 9:361-376

29. Nömayr A, Römer W, Strobel D, Bautz W, Kuwert T (2006) Anatomical accuracy of hybrid SPECT/spiral CT in the lower spine. Nucl Med Commun 27:521-528

30. Han J, Köstler H, Bennewitz C, Kuwert T, Hornegger J (2008) Computer-aided evaluation of anatomical accuracy of image fusion between X-ray CT and SPECT. Comput Med Imaging Graph 32(5):388-395

31. Gilman MD, Fischman AJ, Krishnasetty V, Halpern EF, Aquino SL (2006) Optimal CT breathing protocol for combined thoracic PET/ CT. Am J Roentgenol 187:1357-1360. doi:10.2214/ajr.05.1427

32. Chen J, Caputlu-Wilson S, Shi H, Galt J, Faber T, Garcia E (2006) Automated quality control of emission-transmission misalignment for attenuation correction in myocardial perfusion imaging with SPECT-CT systems. J Nucl Cardiol 13:43-49. doi:10.1016/j.nuclcard.2005.11.007

33. Bockisch A, Freudenberg LS, Schmidt D, Kuwert T (2009) Hybrid imaging by SPECT/CT and PET/CT: proven outcomes in cancer imaging. Semin Nucl Med 39:276-289

34. Mariani G, Bruselli L, Kuwert T, Kim EE, Flotats A, Israel O, Dondi M, Watanabe N (2010) A review on the clinical uses of SPECT/CT. Eur J Nucl Med Mol Imaging 37:1959-1985. doi:10. 1007/s00259-010-1390-8

35. Ritt P, Vija H, Hornegger J, Kuwert T (2011) Absolute quantification in SPECT. Eur J Nucl Med Mol Imaging 38(Suppl 1):S69-S77. doi:10.1007/s00259-011-1770-8

36. Hulme KW, Kappadath SC (2014) Implications of CT noise and artifacts for quantitative ${ }^{99} \mathrm{mTc}$ SPECT/CT imaging. Med Phys 41:042502. doi:10.1118/1.4868511

37. Gnanasegaran G, Cook G, Adamson K, Fogelman I (2009) Patterns, variants, artifacts, and pitfalls in conventional radionuclide bone imaging and SPECT/CT. Semin Nucl Med 39:380-395. doi:10.1053/j.semnuclmed.2009.07.003

38. Bonta DV, Wahl RL (2010) Overcorrection of iodinated contrast attenuation in SPECT-CT: phantom studies. Med Phys 37:4897-4901

39. Goetze S, Wahl RL (2007) Prevalence of misregistration between SPECT and CT for attenuation-corrected myocardial perfusion SPECT. J Nucl Cardiol 14:200-206. doi:10.1016/j.nuclcard.2006. 12.325

40. Wells RG, Soueidan K, Vanderwerf K, Ruddy TD (2012) Comparing slow- versus high-speed CT for attenuation correction of cardiac SPECT perfusion studies. J Nucl Cardiol 19:719-726. doi:10.1007/s12350-012-9555-4

41. Könik A, Kikut J, Lew R, Johnson K, King MA (2013) Comparison of methods of acquiring attenuation maps for cardiac SPECT in the presence of respiratory motion. J Nucl Cardiol 20:1093-1107. doi:10.1007/s12350-013-9791-2 
42. Schleyer PJ, O’Doherty MJ, Barrington SF, Marsden PK (2009) Retrospective data-driven respiratory gating for PET/CT. Phys Med Biol 54:1935-1950. doi:10.1088/0031-9155/54/7/005

43. Kesner AL, Schleyer PJ, Büther F, Walter MA, Schäfers KP, Koo PJ (2014) On transcending the impasse of respiratory motion correction applications in routine clinical imaging - a consideration of a fully automated data driven motion control framework. EJNMMI Phys 1:1-11. doi:10.1186/2197-7364-1-8

44. Kovalski G, Israel O, Keidar Z, Frenkel A, Sachs J, Azhari H (2007) Correction of heart motion due to respiration in clinical myocardial perfusion SPECT scans using respiratory gating. J Nucl Med 48:630-636

45. El Fakhri G, Buvat I, Benali H, Todd-Pokropek A, Di Paola R (2000) Relative impact of scatter, collimator response, attenuation, and finite spatial resolution corrections in cardiac SPECT. J Nucl Med 41:1400-1408

46. Hutton BF, Buvat I, Beekman FJ (2011) Review and current status of SPECT scatter correction. Phys Med Biol 56:R85-R112. doi:10.1088/0031-9155/56/14/r01

47. King MA, Tsui BMW, Pan T-S (1995) Attenuation compensation for cardiac single-photon emission computed tomographic imaging: part 1. Impact of attenuation and methods of estimating attenuation maps. J Nucl Cardiol 2:513-524. doi:10.1016/S10713581(05)80044-3

48. Zaidi H, Koral KF (2004) Scatter modelling and compensation in emission tomography. Eur J Nucl Med Mol Imaging 31:761-782. doi:10.1007/s00259-004-1495-z

49. Jaszczak RJ, Greer KL, Floyd CEJ, Harris CC, Coleman RE (1984) Improved SPECT quantification using compensation for scattered photons. J Nucl Med 25:893-900

50. Ogawa K, Harata Y, Ichihara T, Kubo A, Hashimoto S (1991) A practical method for position-dependent Compton-scatter correction in single photon emission CT. IEEE Trans Med Imaging 10:408-412

51. King MA, DeVries DJ, Pan TS, Pretorius PH, Case JA (1997) An investigation of the filtering of TEW scatter estimates used to compensate for scatter with ordered subset reconstructions. IEEE Trans Nucl Sci 44:1140-1145. doi:10.1109/23.596978

52. Buvat I, Rodriguez-Villafuerte M, Todd-Pokropek A, Benali H, Di Paola R (1995) Comparative assessment of nine scatter correction methods based on spectral analysis using Monte Carlo simulations. J Nucl Med 36:1476-1488

53. Sohlberg A, Watabe H, Iida H (2008) Acceleration of Monte Carlo-based scatter compensation for cardiac SPECT. Phys Med Biol 53:N277-N285. doi:10.1088/0031-9155/53/14/n02

54. Beekman FJ, De Jong HWAM, van Geloven S (2002) Efficient fully 3-D iterative SPECT reconstruction with Monte Carlo-based scatter compensation. IEEE Trans Med Imaging 21:867-877. doi:10.1109/TMI.2002.803130

55. Frey EC, Tsui BMW (1996) A new method for modeling the spatially-variant, object-dependent scatter response function in SPECT. IEEE Nucl Sci Symp Conf Rec 1082-1086. doi:10.1109/ NSSMIC.1996.591559

56. Bruyant PP (2002) Analytic and iterative reconstruction algorithms in SPECT. J Nucl Med 43:1343-1358

57. Shepp LA, Vardi Y (1982) Maximum likelihood reconstruction for emission tomography. IEEE Trans Med Imaging 1:113-122. doi:10.1109/TMI.1982.4307558

58. Lange K, Carson R (1984) EM reconstruction algorithms for emission and transmission tomography. J Comput Assist Tomogr 8:306-316

59. Arosio M, Pasquali C, Crivellaro C, De Ponti E, Morzenti S, Guerra L, Crespi A, Messa C (2011) Performance of a SPECT collimator-detector response reconstruction algorithm: phantom studies and validation in inflammation clinical studies. Q J Nucl Med Mol Imaging 55:671-679
60. Aldridge MD, Waddington WW, Dickson JC, Prakash V, Ell PJ, Bomanji JB (2013) Clinical evaluation of reducing acquisition time on single-photon emission computed tomography image quality using proprietary resolution recovery software. Nucl Med Commun 34:1116-1123. doi:10.1097/MNM.0b013e3283658328

61. Kalantari F, Rajabi H, Saghari M (2012) Quantification and reduction of the collimator-detector response effect in SPECT by applying a system model during iterative image reconstruction: a simulation study. Nucl Med Commun 33:228-238. doi:10.1097/ MNM.0b013e32834e755f

62. Zoccarato O, Scabbio C, De Ponti E, Matheoud R, Leva L, Morzenti S, Menzaghi M, Campini R, Marcassa C, Del Sole A, Garancini S, Crivellaro C, Brambilla M, Lecchi M (2014) Comparative analysis of iterative reconstruction algorithms with resolution recovery for cardiac SPECT studies. A multi-center phantom study. J Nucl Cardiol 21:135-148. doi:10.1007/s12350013-9821-0

63. Druz RS, Phillips LM, Chugkowski M, Boutis L, Rutkin B, Katz S (2011) Wide-beam reconstruction half-time SPECT improves diagnostic certainty and preserves normalcy and accuracy: a quantitative perfusion analysis. J Nucl Cardiol 18:52-61. doi:10. 1007/s12350-010-9304-5

64. Venero CV, Heller GV, Bateman TM, McGhie AI, Ahlberg AW, Katten D, Courter SA, Golub RJ, Case JA, Cullom SJ (2009) A multicenter evaluation of a new post-processing method with depth-dependent collimator resolution applied to full-time and half-time acquisitions without and with simultaneously acquired attenuation correction. J Nucl Cardiol 16:714-725. doi:10.1007/ s12350-009-9106-9

65. Stansfield EC, Sheehy N, Zurakowski D, Vija AH, Fahey FH, Treves ST (2010) Pediatric ${ }^{99} \mathrm{mTc}-\mathrm{MDP}$ bone SPECT with ordered subset expectation maximization iterative reconstruction with isotropic 3D resolution recovery. Radiology 257:793-801. doi:10.1148/radiol.10100102

66. Liu S, Farncombe TH (2007) Collimator-detector response compensation in quantitative SPECT reconstruction. IEEE Nucl Sci Symp Conf Rec 3955-3960. doi:10.1109/NSSMIC.2007.4436983

67. Du Y, Tsui BM, Frey EC (2006) Model-based compensation for quantitative ${ }^{123} \mathrm{I}$ brain SPECT imaging. Phys Med Biol 51:1269-1282. doi:10.1088/0031-9155/51/5/016

68. Se Young C, Fessler JA, Dewaraja YK (2013) Correction for collimator-detector response in SPECT using point spread function template. IEEE Trans Med Imaging 32:295-305. doi:10. 1109/TMI.2012.2225441

69. Kazantsev D, Bousse A, Pedemonte S, Arridge SR, Hutton BF, Ourselin S (2011) Edge preserving bowsher prior with nonlocal weighting for 3D spect reconstruction. IEEE Int Symp Biomed Imaging 1158-1161. doi:10.1109/ISBI.2011.5872607

70. Vija HA (2013) Introduction to XSPECT technology: evolving multi-modal SPECT to become context-based and quantitative. Siemens Medical Solutions USA. http://www.healthcare.siemens. com/molecular-imaging/customer-portal-resource/expand-scan ner-capabilities/symbia-intevo. Accessed 10 October 2014

71. Vija AH, Ma J, Bartenstein P, Froelich J, Kuwert T, Macapinlac H, Mena E, Ilhan H, Yang S, Szabo Z (2014) Reader confidence in lesion detection and lesion characterization with xSPECT Bone. J Nucl Med 55(Suppl 1):1499

72. Lewis DH, Bluestone JP, Savina M, Zoller WH, Meshberg EB, Minoshima S (2006) Imaging cerebral activity in recovery from chronic traumatic brain injury: a preliminary report. J Neuroimaging 16:272-277. doi:10.1111/j.1552-6569.2006.00034.x

73. Sidoti C, Agrillo U (2006) Chronic cortical stimulation for amyotropic lateral sclerosis: a report of four consecutive operated cases after a 2-year follow-up: technical case report. Neurosurgery 58:E384 
74. Shcherbinin S et al (2008) Accuracy of quantitative reconstructions in SPECT/CT imaging. Phys Med Biol 53:4595

75. Du Y et al (2006) Model-based compensation for quantitative ${ }^{123} \mathrm{I}$ brain SPECT imaging. Phys Med Biol 51:1269

76. Vandervoort $E$ et al (2007) Implementation of an iterative scatter correction, the influence of attenuation map quality and their effect on absolute quantitation in SPECT. Phys Med Biol 52:1527

77. Da Silva AJ, Tang HR, Wu MC, Hasegawa BH (1999) Absolute quantitation of myocardial activity in phantoms. IEEE Trans Nucl Sci 46:659-666

78. Zeintl J, Vija AH, Yahil A, Hornegger J, Kuwert T (2010) Quantitative accuracy of clinical ${ }^{99} \mathrm{mTc}$ SPECT/CT using ordered-subset expectation maximization with 3-dimensional resolution recovery, attenuation, and scatter correction. J Nucl Med 51:921-928. doi:10.2967/jnumed.109.071571

79. Cachovan M, Vija A, Hornegger J, Kuwert T (2013) Quantification of ${ }^{99} \mathrm{mTc}-\mathrm{DPD}$ concentration in the lumbar spine with SPECT/CT. EJNMMI Research 3:45
80. Shcherbinin S, Piwowarska-Bilska H, Celler A, Birkenfeld B (2012) Quantitative SPECT/CT reconstruction for ${ }^{177} \mathrm{Lu}$ and ${ }^{177} \mathrm{Lu} /{ }^{90} \mathrm{Y}$ targeted radionuclide therapies. Phys Med Biol 57:5733-5747

81. Beauregard JM, Hofman MS, Pereira JM, Eu P, Hicks RJ (2011) Quantitative $\left({ }^{177}\right) \mathrm{Lu}$ SPECT (QSPECT) imaging using a commercially available SPECT/CT system. Cancer Imaging 11:56-66. doi:10.1102/1470-7330.2011.0012

82. Hapdey S, Soret M, Buvat I (2006) Quantification in simultaneous $\left({ }^{99} \mathrm{~m}\right) \mathrm{Tc} /\left({ }^{123}\right) \mathrm{I}$ brain SPECT using generalized spectral factor analysis: a Monte Carlo study. Phys Med Biol 51:6157-6171. doi:10.1088/0031-9155/51/23/015

83. Bailey DL, Willowson KP (2014) Quantitative SPECT/CT: SPECT joins PET as a quantitative imaging modality.Eur J Nucl Med Mol Imaging 41 Suppl 1:S17-25. doi:10.1007/s00259-013-2542-4

84. Ritt P, Kuwert T (2013) Quantitative SPECT/CT. In: Schober O, Riemann B (eds) Molecular Imaging in Oncology, vol 187., Recent results in cancer researchSpringer, Berlin, pp 313-330 